\title{
Starlikeness and related properties of certain integral operator for multivalent functions
}

\author{
Ram Narayan Mohapatra and Trailokya Panigrahi
}

\begin{abstract}
In this paper, the authors introduce a new general integral operator for multivalent functions. The new sufficient conditions for the operator $\mathcal{J}_{p, \gamma, g}^{\alpha_{i}, \beta_{i}}\left(f_{1}, f_{2}, \ldots, f_{n}\right)$ when $\gamma=1$ is determined for the class of $p$-valently starlike, $p$-valently close-to-convex, uniformly $p$-valent close-to-convex and strongly starlike of order $\delta(0<\delta \leq 1)$ in $\mathbb{U}$. Our results generalize the results of Frasin [7].
\end{abstract}

Mathematics Subject Classification (2010): 30C45.

Keywords: $p$-valent starlike, convex and close-to-convex functions, strongly starlike function, convolution, integral operator.

\section{Introduction and definitions}

Let $\mathcal{A}(p, n)$ denote the class of functions of the form:

$$
f(z)=z^{p}+\sum_{k=n}^{\infty} a_{k+p} z^{k+p} \quad(p, n \in \mathbb{N}:=\{1,2,3 \cdots\})
$$

which are analytic in the open unit disk $\mathbb{U}=\{z: z \in \mathbb{C}$ and $|z|<1\}$. We write $\mathcal{A}(p, 1)=\mathcal{A}_{p}$ and $\mathcal{A}_{1}=\mathcal{A}$.

A function $f \in \mathcal{A}_{p}$ is said to be $p$-valently starlike of order $\delta(0 \leq \delta<p)$, denoted by the class $\mathcal{S}_{p}^{*}(\delta)$ if and only if

$$
\Re\left(\frac{z f^{\prime}(z)}{f(z)}\right)>\delta \quad(z \in \mathbb{U}) .
$$

Also, we say that a function $f \in \mathcal{A}_{p}$ is said to be $p$-valently convex of order $\delta$ $(0 \leq \delta<p)$ and belong to the class $\mathcal{K}_{p}(\delta)$ if and only if

$$
\Re\left(1+\frac{z f^{\prime \prime}(z)}{f^{\prime}(z)}\right)>\delta \quad(z \in \mathbb{U}) .
$$


Further, a function $f \in \mathcal{A}_{p}$ is said to be $p$-valently close-to-convex of order $\delta$ $(0 \leq \delta<p)$, denoted by $\mathcal{C}_{p}(\delta)$ if and only if

$$
\Re\left(\frac{f^{\prime}(z)}{z^{p-1}}\right)>\delta \quad(z \in \mathbb{U}) .
$$

We note that

$$
f(z) \in \mathcal{K}_{p}(\delta) \Longleftrightarrow \frac{z f^{\prime}(z)}{p} \in \mathcal{S}_{p}^{*}(\delta) .
$$

Furthermore, $\mathcal{S}_{p}^{*}(0)=\mathcal{S}_{p}^{*}, \mathcal{K}_{p}(0)=\mathcal{K}_{p}$ and $\mathcal{C}_{p}(0)=\mathcal{C}_{p}$ are respectively denote the class of $p$-valently starlike, $p$-valently convex and $p$-valently close-to-convex functions in $\mathbb{U}$. Also, $\mathcal{S}_{1}^{*}=\mathcal{S}^{*}, \mathcal{K}_{1}=\mathcal{K}$ and $\mathcal{C}_{1}=\mathcal{C}$ are respectively the usual classes of starlike, convex and close-to-convex functions in $\mathbb{U}$.

A function $f \in \mathcal{A}_{p}$ is said to be in the class $\mathcal{U C}_{p}(\beta)$ of uniformly $p$-valent closeto-convex functions of order $\beta(0 \leq \beta<p)$ in $\mathbb{U}$ if and only if

$$
\Re\left(\frac{z f^{\prime}(z)}{g(z)}-\beta\right) \geq\left|\frac{z f^{\prime}(z)}{g(z)}-p\right| \quad(z \in \mathbb{U})
$$

for some $g(z) \in \mathcal{U S}_{p}(\beta)$ where $\mathcal{U S}_{p}(\beta)$ is the class of uniformly $p$-valent starlike functions of order $\beta(-1 \leq \beta<p)$ in $\mathbb{U}$ that satisfies

$$
\Re\left(\frac{z f^{\prime}(z)}{f(z)}-\beta\right) \geq\left|\frac{z f^{\prime}(z)}{f(z)}-p\right| \quad(z \in \mathbb{U}) .
$$

The uniformly $p$-valent starlike functions were first introduced in [9].

For functions $f$ given by $(1.1)$ and $g$ belong to the class $\mathcal{A}_{p}$ given by

$$
g(z)=z^{p}+\sum_{k=n}^{\infty} b_{k+p} z^{k+p} \quad(z \in \mathbb{U})
$$

the Hadamard product (or convolution) of $f$ and $g$ denoted by $f * g$ is given by

$$
(f * g)(z)=z^{p}+\sum_{k=n}^{\infty} a_{k+p} b_{k+p} z^{k+p} \quad(z \in \mathbb{U}) .
$$

Note that for $g(z)=\frac{z^{p}}{1-z}, f * g=f$.

Analogous to the integral operator defined by Goswami and Bulut [10] on pvalent meromorphic functions, we now define the following general integral operator on the space of $p$-valent analytic functions in the class $\mathcal{A}_{p}$.

Definition 1.1. Let $n \in \mathbb{N}, \alpha_{i}, \beta_{i} \in \mathbb{R}_{+} \cup\{0\}$ for all $i=1,2,3, \ldots, n, \gamma \in \mathbb{C}$ with $\Re(\gamma)>0$ and $\alpha=\left(\alpha_{1}, \alpha_{2}, \ldots, \alpha_{n}\right), \beta=\left(\beta_{1}, \beta_{2}, \ldots, \beta_{n}\right)$. For $f_{i}, g \in \mathcal{A}_{p}(1 \leq i \leq n)$, we introduce a new general integral operator $\mathcal{J}_{p, \gamma, g}^{\alpha_{i}, \beta_{i}}: \mathcal{A}_{p}^{n} \longrightarrow \mathcal{A}_{p}$ by

$$
\mathcal{J}_{p, \gamma, g}^{\alpha_{i}, \beta_{i}}\left(f_{1}, f_{2}, . ., f_{n}\right)(z)=\left[\int_{0}^{z} \gamma p t^{\gamma p-1} \prod_{i=1}^{n}\left(\frac{\left(f_{i} * g\right)(t)}{t^{p}}\right)^{\beta_{i}}\left(\frac{\left(f_{i} * g\right)^{\prime}(t)}{p t^{p-1}}\right)^{\alpha_{i}} d t\right]^{\frac{1}{\gamma}}
$$

Here and throughout in the sequel every many-valued function is taken with the principal branch. 
Note that, the integral operator $\mathcal{J}_{p, \gamma, g}^{\alpha_{i}, \beta_{i}}\left(f_{1}, f_{2}, \ldots, f_{n}\right)(z)$ generalizes several previously studied operators as follows:

- For $p=1, g(z)=\frac{z}{1-z}$, we obtain the integral operator

$$
\mathcal{I}_{\gamma}^{\alpha_{i}, \beta_{i}}\left(f_{1}, f_{2}, \ldots, f_{n}\right)(z)=\left\{\int_{0}^{z} \gamma t^{\gamma-1} \prod_{i=1}^{n}\left(f_{i}^{\prime}(t)\right)^{\alpha_{i}}\left(\frac{f_{i}(t)}{t}\right)^{\beta_{i}} d t\right\}^{\frac{1}{\gamma}}
$$

introduced and studied by Frasin [8].

- For $p=1, g(z)=\frac{z}{1-z}$ and $\alpha=(0,0, \ldots, 0)$, we obtain the integral operator

$$
\mathcal{I}_{\gamma}\left(f_{1}, f_{2}, \ldots, f_{n}\right)(z)=\left\{\int_{0}^{z} \gamma t^{\gamma-1} \prod_{i=1}^{n}\left(\frac{f_{i}(t)}{t}\right)^{\beta_{i}} d t\right\}^{\frac{1}{\gamma}}
$$

introduced and studied by Breaz and Breaz [3].

- For $p=1, g(z)=\frac{z}{1-z}, \beta=(0,0, \ldots, 0)$ and $\gamma=1$, we obtain the integral operator $\mathcal{F}_{\alpha_{1}, \alpha_{2}, \ldots, \alpha_{n}}(z)$ where

$$
\mathcal{F}_{\alpha_{1}, \alpha_{2}, \ldots, \alpha_{n}}(z)=\int_{0}^{z} \prod_{i=1}^{n}\left(f_{i}^{\prime}(t)\right)^{\alpha_{i}} d t
$$

introduced and studied by Breaz et al. [4].

- For $p=1, n=1, g(z)=\frac{z}{1-z}, \alpha_{1}=\alpha, \beta_{1}=\beta, f_{1}=f$ and $\gamma=1$, we obtain the integral operator

$$
\mathcal{F}_{\alpha, \beta}(z)=\int_{0}^{z}\left(f^{\prime}(t)\right)^{\alpha}\left(\frac{f(t)}{t}\right)^{\beta} d t
$$

studied in [5].

- For $p=1, n=1, g(z)=\frac{z}{1-z}, \alpha_{1}=0, \beta_{1}=\beta, f_{1}=f$ and $\gamma=1$, we obtain the integral operator

$$
\mathcal{F}_{\beta}(z)=\int_{0}^{z}\left(\frac{f(t)}{t}\right)^{\beta} d t
$$

studied in [11]. In particular, for $\beta=1$, the above operator reduces to

$$
\mathcal{I}(z)=\int_{0}^{z} \frac{f(t)}{t} d t
$$

known as Alexander integral operator (see [1]).

- For $p=1, n=1, g(z)=\frac{z}{1-z}, \beta_{1}=0, \alpha_{1}=\alpha, f_{1}=f$ and $\gamma=1$, we obtain the integral operator

studied in [15] (also see [16]).

$$
\mathcal{G}_{\alpha}(z)=\int_{0}^{z}\left(f^{\prime}(t)\right)^{\alpha} d t
$$

- For $g(z)=\frac{z^{p}}{1-z}, \alpha=(0,0, \ldots, 0)$ and $\gamma=1$, we obtain the integral operator

$$
\mathcal{F}_{p}(z)=\int_{0}^{z} p t^{p-1} \prod_{i=1}^{n}\left(\frac{f_{i}(t)}{t^{p}}\right)^{\beta_{i}} d t
$$


introduced and studied by Frasin [7].

- For $g(z)=\frac{z^{p}}{1-z}, \beta=(0,0, \ldots, 0)$ for $i=1,2,3, . ., n$ and $\gamma=1$, we obtain the integral operator

$$
\mathcal{G}_{p}(z)=\int_{0}^{z} p t^{p-1} \prod_{i=1}^{n}\left(\frac{f_{i}^{\prime}(t)}{p t^{p-1}}\right)^{\alpha_{i}} d t
$$

introduced and studied by Frasin [7].

Various sufficient conditions for convexity and starlikeness of multivalent functions corresponding to different integral operators have been obtained by various authors. Motivated by the aforementioned work, in this paper the authors derive various sufficient conditions for the operator defined in (1.7) when $\gamma=1$ to be $p$-valently starlike, $p$-valently close-to-convex, uniformly close-to-convex and strongly starlike of order $\delta(0<\delta \leq 1)$ in $\mathbb{U}$.

\section{Preliminaries}

In order to derive our main results, we need the following lemmas.

Lemma 2.1. (see [12]) If $f \in \mathcal{A}_{p}$ satisfies

$$
\Re\left\{1+\frac{z f^{\prime \prime}(z)}{f^{\prime}(z)}\right\}<p+\frac{1}{4} \quad(z \in \mathbb{U}),
$$

then $f$ is p-valently starlike in $\mathbb{U}$.

Lemma 2.2. (see [2]) If $f \in \mathcal{A}_{p}$ satisfies

$$
\Re\left(1+\frac{z f^{\prime \prime}(z)}{f^{\prime}(z)}\right)<p+\frac{1}{3} \quad(z \in \mathbb{U}),
$$

then $f$ is uniformly p-valent close-to-convex in $\mathbb{U}$.

Lemma 2.3. (see [17]) If $f \in \mathcal{A}_{p}$ satisfies

$$
\Re\left\{1+\frac{z f^{\prime \prime}(z)}{f^{\prime}(z)}\right\}<p+\frac{a+b}{(1+a)(1-b)} \quad(z \in \mathbb{U}),
$$

where $a>0, b \geq 0$ and $a+2 b \leq 1$, then $f$ is p-valently close-to-convex in $\mathbb{U}$.

Lemma 2.4. (see [14]) If $f \in \mathcal{A}_{p}$ satisfies

$$
\Re\left\{1+\frac{z f^{\prime \prime}(z)}{f^{\prime}(z)}\right\}>\frac{p}{4}-1 \quad(z \in \mathbb{U}),
$$

then

$$
\Re \sqrt{\frac{z f^{\prime}(z)}{f(z)}}>\frac{\sqrt{p}}{2} \quad(z \in \mathbb{U})
$$


Lemma 2.5. (see [13]) If $f \in \mathcal{A}_{p}$ satisfies

$$
\Re\left\{1+\frac{z f^{\prime \prime}(z)}{f^{\prime}(z)}\right\}>p-\frac{\delta}{2} \quad(z \in \mathbb{U}),
$$

then

$$
\left|\arg \frac{z f^{\prime}(z)}{f(z)}\right|<\frac{\pi}{2} \delta \quad(0<\delta \leq 1 ; z \in \mathbb{U}),
$$

or $f$ is strongly starlike of order $\delta$ in $\mathbb{U}$.

Lemma 2.6. (see [6]) If $f \in \mathcal{A}_{p}$ satisfies

$$
\left|\frac{z f^{\prime \prime}(z)}{f^{\prime}(z)}+1-p\right|<p+1 \quad(z \in \mathbb{U})
$$

then $f$ is p-valently starlike in $\mathbb{U}$.

\section{Main results}

In this section, we investigate sufficient conditions for the integral operator $\mathcal{J}_{p, 1, g}^{\alpha_{i}, \beta_{i}}\left(f_{1}, f_{2}, \ldots, f_{n}\right)(z)$ to be in the class $\mathcal{S}_{p}^{*}$. For the sake of simplicity, we shall write $\mathcal{J}_{p, g}(z)$ instead of $\mathcal{J}_{p, 1, g}^{\alpha_{i}, \beta_{i}}\left(f_{1}, f_{2}, \ldots, f_{n}\right)(z)$.

Theorem 3.1. Let $\alpha_{i}, \beta_{i} \in \mathbb{R}_{+} \cup\{0\}$ for all $i=1,2,3, \ldots, n, \alpha=\left(\alpha_{1}, \alpha_{2}, \ldots, \alpha_{n}\right)$ and $\beta=\left(\beta_{1}, \beta_{2}, \ldots, \beta_{n}\right)$. If $f_{i}, g \in \mathcal{A}_{p}(1 \leq i \leq n)$ satisfies

$$
\Re\left\{\alpha_{i} \frac{z\left(f_{i} * g\right)^{\prime \prime}(z)}{\left(f_{i} * g\right)^{\prime}(z)}+\beta_{i} \frac{z\left(f_{i} * g\right)^{\prime}(z)}{\left(f_{i} * g\right)(z)}\right\}<(p-1) \alpha_{i}+p \beta_{i}+\frac{1}{4 n} \quad(z \in \mathbb{U}),
$$

then the general integral operator $\mathcal{J}_{p, g}(z) \in \mathcal{S}_{p}^{*}$.

Proof. From (1.7), it is easy to see that

$$
\begin{aligned}
\mathcal{J}_{p, g}^{\prime}(z)=p z^{p-1} & \left(\frac{\left(f_{1} * g\right)^{\prime}(z)}{p z^{p-1}}\right)^{\alpha_{1}}\left(\frac{\left(f_{1} * g\right)(z)}{z^{p}}\right)^{\beta_{1}} \ldots \\
& \left(\frac{\left(f_{n} * g\right)^{\prime}(z)}{p z^{p-1}}\right)^{\alpha_{n}}\left(\frac{\left(f_{n} * g\right)(z)}{z^{p}}\right)^{\beta_{n}} .
\end{aligned}
$$

Differentiating (3.2) logarithmically with respect to $z$ and multiply by $z$, we obtain

$$
\frac{z \mathcal{J}_{p, g}^{\prime \prime}(z)}{\mathcal{J}_{p, g}^{\prime}(z)}=(p-1)+\sum_{i=1}^{n} \alpha_{i}\left(\frac{z\left(f_{i} * g\right)^{\prime \prime}(z)}{\left(f_{i} * g\right)^{\prime}(z)}-p+1\right)+\sum_{i=1}^{n} \beta_{i}\left(\frac{z\left(f_{i} * g\right)^{\prime}(z)}{\left(f_{i} * g\right)(z)}-p\right),
$$

which implies

$$
1+\frac{z \mathcal{J}_{p, g}^{\prime \prime}(z)}{\mathcal{J}_{p, g}^{\prime}(z)}=\sum_{i=1}^{n}\left(\alpha_{i} \frac{z\left(f_{i} * g\right)^{\prime \prime}(z)}{\left(f_{i} * g\right)^{\prime}(z)}+\beta_{i} \frac{z\left(f_{i} * g\right)^{\prime}(z)}{\left(f_{i} * g\right)(z)}\right)+p-\sum_{i=1}^{n}\left[(p-1) \alpha_{i}+p \beta_{i}\right]
$$


Taking real part on both sides of $(3.3)$, we get

$$
\begin{array}{r}
\Re\left(1+\frac{z \mathcal{J}_{p, g}^{\prime \prime}(z)}{\mathcal{J}_{p, g}^{\prime}(z)}\right)=p-\sum_{i=1}^{n}\left[(p-1) \alpha_{i}+p \beta_{i}\right]+ \\
\sum_{i=1}^{n} \Re\left(\alpha_{i} \frac{z\left(f_{i} * g\right)^{\prime \prime}(z)}{\left(f_{i} * g\right)^{\prime}(z)}+\beta_{i} \frac{z\left(f_{i} * g\right)^{\prime}(z)}{\left(f_{i} * g\right)(z)}\right) .
\end{array}
$$

Using (3.1) in (3.4) yields

$$
\Re\left(1+\frac{z \mathcal{J}_{p, g}^{\prime \prime}(z)}{\mathcal{J}_{p, g}^{\prime}(z)}\right)<p+\frac{1}{4} \quad(z \in \mathbb{U}) .
$$

Hence by Lemma $2.1, \mathcal{J}_{p, g}(z)$ is $p$-valently starlike in $\mathbb{U}$ which implies $\mathcal{J}_{p, g}(z) \in \mathcal{S}_{p}^{*}$. This complete the proof of Theorem 3.1.

Taking $\alpha=(0,0, \ldots, 0)$ and $g(z)=\frac{z^{p}}{1-z}(z \in \mathbb{U})$ in Theorem 3.1, we get the following result.

Corollary 3.2. ([7], Theorem 2.1) Let $\beta_{i}>0$ be real numbers for all $i=1,2,3, \ldots, n$. If $f_{i} \in \mathcal{A}_{p}$ for all $i=1,2,3, . ., n$ satisfies

$$
\Re\left(\frac{z f_{i}^{\prime}(z)}{f_{i}(z)}\right)<p+\frac{1}{4 \sum_{i=1}^{n} \beta_{i}} \quad(z \in \mathbb{U})
$$

then $\mathcal{F}_{p}$ is p-valently starlike in $\mathbb{U}$.

Remark 3.3. If we set $n=p=1, \beta_{1}=\beta, f_{1}=f$ in Corollary 3.2, then we have $([7]$, Corollary 2.2).

Further, taking $\beta=(0,0, \ldots, 0) g(z)=\frac{z^{p}}{1-z}$ in Theorem 3.1 we get the following result.

Corollary 3.4. ([7], Theorem 3.1) Let $\alpha_{i}>0$ be real numbers for all $i=1,2,3, \ldots, n$. If $f_{i} \in \mathcal{A}_{p}$ for all $i=1,2,3, \ldots, n$ satisfies

$$
\Re\left(1+\frac{z f_{i}^{\prime \prime}(z)}{f_{i}^{\prime}(z)}\right)<p+\frac{1}{4 \sum_{i=1}^{n} \alpha_{i}} \quad(z \in \mathbb{U}),
$$

then $\mathcal{G}_{p}$ is p-valently starlike in $\mathbb{U}$.

Remark 3.5. Letting $n=p=1, \alpha_{1}=\alpha$ and $f_{1}=f$ in Corollary 3.4 we get the following result due to Frasin ([7], Corollary 3.2).

Furthermore, taking $p=1, n=1, \alpha_{1}=\alpha, \beta_{1}=\beta, f_{1}=f$ and $g(z)=\frac{z}{1-z}$ in Theorem 3.1, we have the following:

Corollary 3.6. Let $f \in \mathcal{A}$ and $\alpha, \beta>0$. If

$$
\Re\left\{\alpha \frac{z f^{\prime \prime}(z)}{f^{\prime}(z)}+\beta \frac{z f^{\prime}(z)}{f(z)}\right\}<\beta+\frac{1}{4} \quad(z \in \mathbb{U})
$$

then the integral operator $\mathcal{F}_{\alpha, \beta}(z)$ defined in (1.11) belong to starlike function class $\mathcal{S}_{p}^{*}$.

The next theorem gives another sufficient condition for the integral operator $\mathcal{J}_{p, g}$ to be $p$-valently starlike functions in $\mathcal{U}$. 
Theorem 3.7. Let $\alpha_{i}, \beta_{i} \in \mathbb{R}_{+} \cup\{0\}$ for all $i=1,2, \ldots, n, \alpha=\left(\alpha_{1}, \alpha_{2}, \ldots, \alpha_{n}\right)$ and $\beta=\left(\beta_{1}, \beta_{2}, \ldots, \beta_{n}\right)$. If $f_{i}, g \in \mathcal{A}_{p}$ for all $i=1,2, \ldots, n$ satisfies the relation

$$
\left|\alpha_{i} \frac{z\left(f_{i} * g\right)^{\prime \prime}(z)}{\left(f_{i} * g\right)^{\prime}(z)}+\beta_{i}\left(\frac{z\left(f_{i} * g\right)^{\prime}(z)}{\left(f_{i} * g\right)(z)}-p\right)\right|<\frac{p+1}{n}-(p-1) \alpha_{i} \quad(z \in \mathbb{U})
$$

where $\sum_{i=1}^{n} \alpha_{i}>1$, then $\mathcal{J}_{p, g}$ is p-valently starlike in $\mathcal{U}$.

Proof. From (3.3) and applications of triangle's inequalities give

$$
\begin{array}{r}
\left|1+\frac{z \mathcal{J}_{p, g}^{\prime \prime}(z)}{\mathcal{J}_{p, g}^{\prime}(z)}-p\right|=\mid \sum_{i=1}^{n}\left(\alpha_{i} \frac{z\left(f_{i} * g\right)^{\prime \prime}(z)}{\left(f_{i} * g\right)^{\prime}(z)}+\beta_{i} \frac{z\left(f_{i} * g\right)^{\prime}(z)}{\left(f_{i} * g\right)(z)}\right)- \\
\sum_{i=1}^{n}\left((p-1) \alpha_{i}+p \beta_{i}\right)|=| \sum_{i=1}^{n}\left(\alpha_{i} \frac{z\left(f_{i} * g\right)^{\prime \prime}(z)}{\left(f_{i} * g\right)^{\prime}(z)}+\beta_{i}\left(\frac{z\left(f_{i} * g\right)^{\prime}(z)}{\left(f_{i} * g\right)(z)}-p\right)\right)- \\
(p-1) \sum_{i=1}^{n} \alpha_{i}\left|<\sum_{i=1}^{n}\right| \alpha_{i} \frac{z\left(f_{i} * g\right)^{\prime \prime}(z)}{\left(f_{i} * g\right)^{\prime}(z)}+\beta_{i}\left(\frac{z\left(f_{i} * g\right)^{\prime}(z)}{\left(f_{i} * g\right)(z)}-p\right) \mid+(p-1) \sum_{i=1}^{n} \alpha_{i}
\end{array}
$$

Making use of (3.6) in (3.7)we get

$$
\left|1+\frac{z \mathcal{J}_{p, g}^{\prime \prime}(z)}{\mathcal{J}_{p, g}^{\prime}(z)}-p\right|<p+1
$$

Therefore, the result follows by application of Lemma 2.6. The proof of Theorem 3.7 is completed.

Remark 3.8. Putting $g(z)=\frac{z^{p}}{1-z}, \alpha=(0,0, \ldots, 0)$ in Theorem 3.7 we get the result of Frasin ([7], Theorem 2.3).

Remark 3.9. Putting $g(z)=\frac{z^{p}}{1-z}, \beta=(0,0, \ldots, 0)$ in Theorem 3.7 we get the result of Frasin ([7], Theorem 3.3).

Remark 3.10. Putting $n=1, p=1, \alpha_{1}=0, f_{1}=f, \beta_{1}=\beta>0, g(z)=\frac{z}{1-z}$ in Theorem 3.7 we get the result of Frasin ([7], Corollary 2.4).

Remark 3.11. Letting $n=1, p=1, \alpha_{1}=\alpha>0, f_{1}=f, \beta=(0,0, \ldots, 0), g(z)=\frac{z}{1-z}$ in Theorem 3.7 we get the result of Frasin ([7], Corollary 3.4).

\section{Close-to-convex function}

The following theorem gives sufficient conditions for the integral operator $\mathcal{J}_{p, g}$ to be $p$-valently close-to-convex in $\mathbb{U}$.

Theorem 4.1. Let $f_{i}, g \in \mathcal{A}_{p}, \alpha_{i}, \beta_{i} \in \mathbb{R}_{+} \cup\{0\}$ for all $i=1,2,3, \ldots, n, \alpha=$ $\left(\alpha_{1}, \alpha_{2}, \ldots, \alpha_{n}\right)$ and $\beta=\left(\beta_{1}, \beta_{2}, \ldots, \beta n\right)$. For $z \in \mathbb{U}$, if

$$
\Re\left\{\alpha_{i} \frac{z\left(f_{i} * g\right)^{\prime \prime}(z)}{\left(f_{i} * g\right)^{\prime}(z)}+\beta_{i} \frac{z\left(f_{i} * g\right)^{\prime}(z)}{\left(f_{i} * g\right)(z)}\right\}<(p-1) \alpha_{i}+p \beta_{i}+\frac{a+b}{n(1+a)(1-b)},
$$

where $a>0, b \geq 0$ and $a+2 b \leq 1$, then $\mathcal{J}_{p, g}(z)$ is p-valently close-to-convex function in $\mathbb{U}$. 
Proof. Making use of (4.1) in (3.3), we get

$$
\Re\left(1+\frac{z \mathcal{J}_{p, g}^{\prime \prime}(z)}{\mathcal{J}_{p, g}^{\prime}(z)}\right)<p+\frac{a+b}{(1+a)(1-b)} .
$$

Hence by Lemma 2.3 we conclude that $\mathcal{J}_{p, g} \in \mathcal{C}_{p}(\delta)$.

Remark 4.2. Putting $g(z)=\frac{z^{p}}{1-z}, \alpha=(0,0,0, \ldots, 0)$ and $\beta_{i}>0$ in Theorem 4.1, we get the result due to Frasin ([7], Theorem 2.5).

Remark 4.3. Putting $g(z)=\frac{z^{p}}{1-z}, \beta=(0,0,0, \ldots, 0)$ and $\alpha_{i}>0$ in Theorem 4.1, we get the result due to Frasin ([7], Theorem 3.5).

Remark 4.4. Letting $n=p=1, \alpha_{1}=0, \beta_{1}=\beta>0, f_{1}=f$ and $g(z)=\frac{z}{1-z}$ in Theorem 4.1 we get the result due to Frasin ([7], Corollary 2.6).

Remark 4.5. Letting $n=p=1, \beta_{1}=0, \alpha_{1}=\alpha, f_{1}=f$ and $g(z)=\frac{z}{1-z}$ in Theorem 4.1 we get the result due to Frasin ([7], Corollary 3.6).

\section{Uniformly close-to-convex function}

In this section we give sufficient conditions for the generalize integral operator $\mathcal{J}_{p, g}(z)$ to be uniformly close-to-convex in $\mathbb{U}$.

Theorem 5.1. Let $f_{i}, g \in \mathcal{A}_{p}, \alpha_{i}, \beta_{i} \in \mathbb{R}_{+} \cup\{0\}$ for all $i=1,2,3, \ldots, n$ and $\alpha=$ $\left(\alpha_{1}, \alpha_{2}, \ldots, \alpha_{n}\right)$ and $\beta=\left(\beta_{1}, \beta_{2}, \ldots, \beta_{n}\right)$. If

$$
\Re\left(\alpha_{i} \frac{z\left(f_{i} * g\right)^{\prime \prime}(z)}{\left(f_{i} * g\right)^{\prime}(z)}+\beta_{i} \frac{z\left(f_{i} * g\right)^{\prime}(z)}{\left(f_{i} * g\right)(z)}\right)<(p-1) \alpha_{i}+p \beta_{i}+\frac{1}{3 n} \quad(z \in \mathbb{U}),
$$

then $\mathcal{J}_{p, g}(z)$ is uniformly p-valent close-to-convex in $\mathbb{U}$.

Proof. Making using (5.1) in (3.3) and an application of Lemma 2.2 give the result. The proof of Theorem 5.1 is thus completed.

Remark 5.2. Putting $g(z)=\frac{z^{p}}{1-z}, \beta_{i}>0$ and $\alpha=(0,0, \ldots, 0)$ in Theorem 5.1 we get the frasin ([7], Theorem 2.7).

Remark 5.3. Letting $g(z)=\frac{z^{p}}{1-z}, \beta=(0,0, . ., 0)$ and $\alpha_{i}>0$ for $i=1,2,3, . ., n$ in Theorem 5.1 we get the result of Frasin ([7], Theorem 3.7).

Remark 5.4. Taking $n=p=1, \beta_{1}=\beta$ and $f_{1}=f$ in Remark 5.2 we have ([7], Corollary 2.8).

Remark 5.5. Taking $n=p=1, \alpha_{1}=\alpha$ and $f_{1}=f$ in Remark 5.3 we have ([7], Corollary 3.8). 


\section{Strong starlikeness of the operators $\mathcal{J}_{p, g}$}

The following theorem gives sufficient conditions for the operator $\mathcal{J}_{p, g}$ to be strongly starlike of order $\delta$ in $\mathbb{U}$.

Theorem 6.1. Let $\alpha_{i}, \beta_{i} \in \mathbb{R}_{+} \cup\{0\}$ for all $i=1,2,3, \ldots, n, \alpha=\left(\alpha_{1}, \alpha_{2}, \ldots, \alpha_{n}\right)$ and $\beta=\left(\beta_{1}, \beta_{2}, \ldots, \beta_{n}\right)$. If $f_{i}, g \in \mathcal{A}_{p}$ for all $i=1,2,3, \ldots, n$ satisfies

$$
\Re\left\{\alpha_{i} \frac{z\left(f_{i} * g\right)^{\prime \prime}(z)}{\left(f_{i} * g\right)^{\prime}(z)}+\beta_{i} \frac{z\left(f_{i} * g\right)^{\prime}(z)}{\left(f_{i} * g\right)(z)}\right\}>(p-1) \alpha_{i}+p \beta_{i}-\frac{\delta}{2 n} \quad(z \in \mathbb{U}),
$$

then $\mathcal{J}_{p, g}$ is strongly starlike of order $\delta(0<\delta \leq 1)$ in $\mathbb{U}$.

Proof. In view of (3.3) and (6.1) and by using Lemma 2.5, we deduce that $\mathcal{J}_{p, g}$ is strongly starlike of order $\delta$.

Putting $g(z)=\frac{z^{p}}{1-z}, \alpha=(0,0, \ldots, 0)$ in Theorem 6.1 , we get the following result:

Corollary 6.2. ([7], Theorem 4.1) Let $\beta_{i}>0$ be real numbers for all $i=1,2,3, . ., n$. If $f_{i} \in \mathcal{A}_{p}$ for all $i=1,2,3, . ., n$ satisfies

$$
\Re\left(\frac{z f_{i}^{\prime}(z)}{f_{i}(z)}\right)>p-\frac{\delta}{2 \sum_{i=1}^{n} \beta_{i}} \quad(z \in \mathbb{U}),
$$

then $\mathcal{F}_{p}$ is strongly starlike of order $\delta(0<\delta \leq 1)$ in $\mathbb{U}$.

Remark 6.3. Putting $n=p=1, \beta_{1}=\beta$ and $f_{1}=f$ in the Corollary 6.2 , we get the result of Frasin ([7], Corollary 4.2).

Further, letting $g(z)=\frac{z^{p}}{1-z}, \beta=(0,0,0, \ldots, 0)$ in Theorem 6.1 we get the following result.

Corollary 6.4. ([7], Theorem 4.3) Let $\alpha_{i}>0$ be the real numbers for all $i=1,2,3, \ldots, n$. If $f_{i} \in \mathcal{A}_{p}$ for $i=1,2,3, . ., n$ satisfies

$$
\Re\left(1+\frac{z f_{i}^{\prime \prime}(z)}{f_{i}^{\prime}(z)}\right)>p-\frac{\delta}{2 \sum_{i=1}^{n} \alpha_{i}} \quad(z \in \mathbb{U}),
$$

then $\mathcal{G}_{p}$ is strongly starlike of order $\delta 0<\delta \leq 1$ in $\mathbb{U}$.

Remark 6.5. Letting $n=p=1, \alpha_{1}=\alpha>0$ and $f_{1}=f$ in the above Corollary, we get the result (see [7], Corollary 4.4).

Theorem 6.6. Let $\alpha_{i}, \beta_{i} \in \mathbb{R}_{+} \cup\{0\}$ for all $i=1,2,3, \ldots, n, \alpha=\left(\alpha_{1}, \alpha_{2}, \ldots, \alpha_{n}\right)$ and $\beta=\left(\beta_{1}, \beta_{2}, \ldots, \beta_{n}\right)$. If $f_{i}, g \in \mathcal{A}_{p}$ for all $i=1,2,3, \ldots, n$ satisfies

$$
\Re\left\{\alpha_{i} \frac{z\left(f_{i} * g\right)^{\prime \prime}(z)}{\left(f_{i} * g\right)^{\prime}(z)}+\beta_{i} \frac{z\left(f_{i} * g\right)^{\prime}(z)}{\left(f_{i} * g\right)(z)}\right\}>(p-1) \alpha_{i}+p \beta_{i}-\frac{3 p+4}{4 n} \quad(z \in \mathbb{U}),
$$

then

$$
\Re \sqrt{\frac{z \mathcal{J}_{p, g}^{\prime}(z)}{\mathcal{J}_{p, g}(z)}}>\frac{\sqrt{p}}{2}
$$


Proof. Using (6.4) in (3.4), we have

$$
\Re\left(1+\frac{z \mathcal{J}_{p, g}^{\prime \prime}(z)}{\mathcal{J}_{p, g}(z)}\right)>\frac{p}{4}-1 .
$$

The result follows in view of Lemma 2.4.

Remark 6.7. Putting $g(z)=\frac{z^{p}}{1-z}, \alpha=(0,0, \ldots, 0)$ in the Theorem 6.6 , we get the result of Frasin ([7],Theorem 2.9)

Remark 6.8. Putting $n=p=1, \beta_{1}=1$ and $f_{1}=f, \alpha=(0,0, \ldots, 0)$ and $g(z)=\frac{z}{1-z}$ in the Theorem 6.6, we get the result of Frasin ([7],Corollary 2.10).

Remark 6.9. Taking $\beta=(0,0, \ldots, 0)$ and $g(z)=\frac{z^{p}}{1-z}$ in the Theorem 6.6 , we get the result of Frasin ([7], Theorem 3.9).

Remark 6.10. Letting $n=p=1, \alpha_{1}=1$ and $f_{1}=f, \beta=(0,0, \ldots, 0)$ and $g(z)=\frac{z}{1-z}$ in the Theorem 6.6, we get the result of Frasin ([7], Corollary 3.10).

Acknowledgement. The authors thank the reviewer for many useful suggestions for revision which improved the content of the manuscript.

\section{References}

[1] Alexander, W., Functions which map the interior of the unit circle upon simple regions, Ann. Math., 17(1915), no. 1, 12-22.

[2] Al-Kharsani, H.A., Al-Hajiry, S.S., A note on certain inequalities for p-valent functions, J. Inequal. Pure Appl. Math., 9(2008), no. 3.

[3] Breaz, D., Breaz, N., Two integral operators, Stud. Univ. Babeş-Bolyai Math., ClujNapoca, 3(2002), 13-19.

[4] Breaz, D., Owa, S., Breaz, N., A new integral univalent operator, Acta Univ. Apul., 16(2008), 11-16.

[5] Dorf, M., Szynal, J., Linear invariance and integral operators of univalent functions, Demonstratio Math., 38(2005), no. 1, 47-57.

[6] Dziok, J., Applications of the Jack lemma, Acta Math. Hungar., 105(2004), no. 1-2, 93-102.

[7] Frasin, B.A., New general integral operators of p-valent functions, J. Inequal. Pure Appl. Math., 10(2009), no. 4.

[8] Frasin, B.A., Order of convexity and univalency of general integral operator, J. Franklin Institute, 348(2011), 1013-1019.

[9] Goodman, A.W., On uniformly starlike functions, J. Math. Anal. Appl., 55(1991), 364370.

[10] Goswami, P., Bulut, S., Starlikeness of general integral operator for meromorphic multivalent functions, J. Complex Anal., Vol. 2013, Art. ID 690584.

[11] Miller, S.S., Mocanu, P.T., Reade, M.O., Starlike integral operators, Pacific J. Math., 79(1978), no. 1, 157-168.

[12] Nunokawa, M., On the multivalent functions, Indian J. Pure Appl. Math., 20(1989), 577-582. 
[13] Nunokawa, M., Owa, S., Ikeda, A., On the strongly starlikeness of multivalently convex functions of order $\alpha$, IJMMS, 28(2001), no. 1, 51-55.

[14] Owa, S., On the Nunokawa's conjecture for multivalent functions, Bull. Austral. Math. Soc., 41(1990), 301-305.

[15] Pascu, N., Pescar, V., On the integral operators of Kim-Merkis and Pfaltzgraff, Mathematica, 32(1990), no. 55, 185-192.

[16] Pfaltzgraff, J.A., Univalence of the integral of $f^{\prime}(z)^{\lambda}$, Bull. London Math. Soc., 7(1975), no. 3, 254-256.

[17] Raina, R.K., Bapna, I.B., Inequalities defining certain subclasses of analytic functions involving fractional calculus operators, J. Inequal. Pure Appl. Math., 5(2004), no. 2.

Ram Narayan Mohapatra

Department of Mathematics

University of Central Florida

Orlando, FL. 32816, USA

e-mail: ram.mohapatra@ucf.edu

Trailokya Panigrahi

Department of Mathematics

School of Applied Sciences, KIIT University

Bhubaneswar-751024, Orissa, India

e-mail: trailokyap6@gmail.com 